\title{
Experimental characterization of the frequency modulation behavior of vertical cavity surface emitting lasers
}

Jia Chen, Andreas Hangauer, and Rainer StrzodaMarkus-Christian Amann

Citation: Appl. Phys. Lett. 91, 141105 (2007); doi: 10.1063/1.2794406

View online: http://dx.doi.org/10.1063/1.2794406

View Table of Contents: http://aip.scitation.org/toc/apl/91/14

Published by the American Institute of Physics

This article may be downloaded for personal use only. Any other use requires prior permission of the author and AIP Publishing. This article appeared in Appl. Phys. Lett. 91, 141105 (2007), doi: 10.1063/1.2794406, and may be found at https://aip.scitation.org/doi/10.1063/1.2794406.

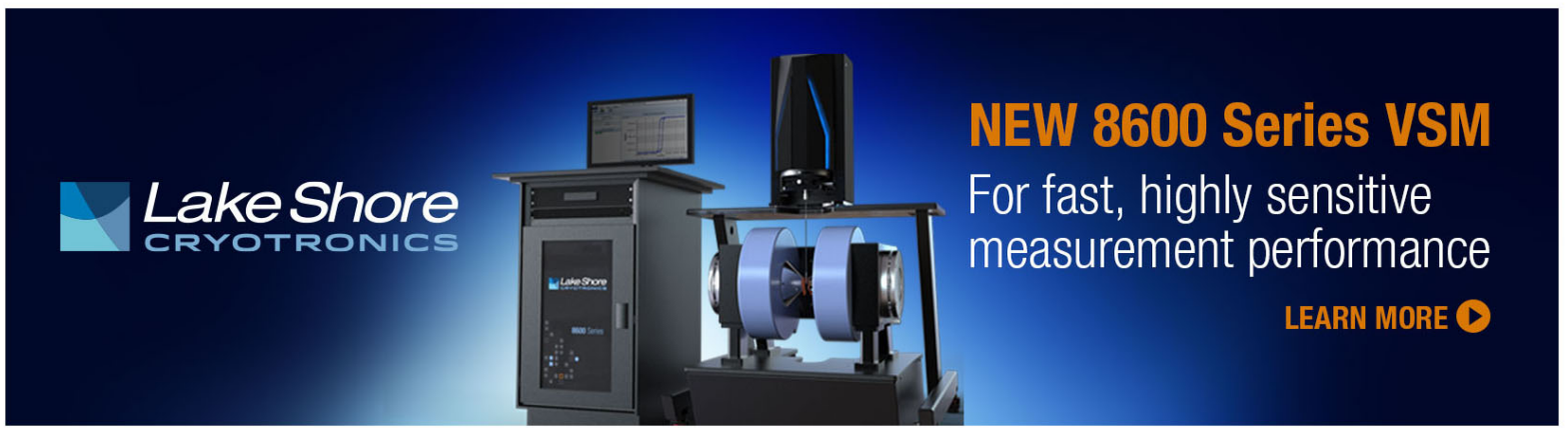




\title{
Experimental characterization of the frequency modulation behavior of vertical cavity surface emitting lasers
}

\author{
Jia Chen, ${ }^{\text {a) }}$ Andreas Hangauer, and Rainer Strzoda \\ Power and Sensor Systems, Siemens Corporate Technology, Otto-Hahn-Ring 6, D-81739 Munich, Germany \\ Markus-Christian Amann \\ Walter Schottky Institute, Technical University of Munich, Am Coulombwall 3, D-85748 Garching, Germany
}

(Received 23 July 2007; accepted 14 September 2007; published online 1 October 2007)

\begin{abstract}
The frequency response of the current-to-wavelength tuning rate (FM response) was measured for two different vertical cavity surface emitting lasers (VCSELs) up to frequencies of $1 \mathrm{MHz}$ : GaAs-based VCSEL $(763 \mathrm{~nm})$ and InP-based VCSEL $(1853 \mathrm{~nm})$. Both of them show the same qualitative FM response behavior, which can be described by a square root law and therefore cannot be modeled by a first order low pass. The square root law behavior is a significant advantage for laser spectroscopy applications with VCSELs because the decrease of the current-to-wavelength tuning coefficient is less severe as in the case of the first order low pass. (C) 2007 American Institute of Physics. [DOI: 10.1063/1.2794406]
\end{abstract}

Vertical cavity surface emitting lasers (VCSELs) have gained increasing importance for numerous optoelectronic applications because of their intrinsic advantages, such as low threshold current and low fabrication cost. ${ }^{1,2}$ While short wavelength VCSELs $(<1 \mu \mathrm{m})$ have been available for more than a decade, ${ }^{2-4}$ also high-performance InP-based long wavelength $(1.3-2.05 \mu \mathrm{m})$ VCSELs have recently been developed. ${ }^{5,6}$ As with edge emitting lasers, VCSELs allow tuning of the wavelength via the laser current. Up to several megahertz, the tuning of the wavelength is basically a thermal effect: the current causes heating of the VCSEL chip, and the resulting increase in refractive index of the material increases the laser wavelength. ${ }^{7}$ An additional effect that changes the wavelength is the plasma effect, which describes the influence of the carrier density on the wavelength. ${ }^{8}$ In the case of a directly modulated VCSEL, the plasma effect is several orders of magnitudes lower than the thermal effect at frequencies up to at least $10 \mathrm{MHz}$. For small current variations around a bias point, the differential current to wavelength shift behavior is linear. The laser behavior can be considered linear and time invariant with respect to its frequency/wavelength modulation (FM) behavior, which means that a small sinusoidal current variation always results in a sinusoidal wavelength variation. The tuning coefficient given by the ratio of the amplitudes is frequency dependent, and it is obvious that the laser will have a kind of low-pass behavior since heat conduction is an inert process. In the following, the frequency response of the current-towavelength tuning rate is defined as "FM response." The FM response of a laser is of considerable interest for wavelength modulation spectroscopy (WMS) applications, because in this application the wavelength of the emitting light is modulated sinusoidally through the injection current. It is important to know how the current to wavelength efficiency depends on the modulation frequency. For distributed feedback (DFB) lasers/edge emitting lasers, a theory of the FM response $^{8}$ and also measurements ${ }^{9}$ already exist. In this paper, measurements of the FM response of two different kinds

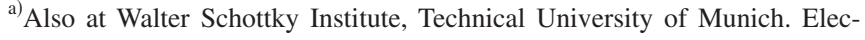
tronic mail: jia.chen@wsi.tum.de of VCSELs are presented: InP-based $1853 \mathrm{~nm}$ VCSEL (VERTILAS) and GaAs-based $763 \mathrm{~nm}$ VCSEL (ULM Photonics). Compared to existing measurements for DFB lasers up to $100 \mathrm{kHz},{ }^{9}$ we take more measurement points around cutoff frequency and are able to measure at frequencies up to $1 \mathrm{MHz}$. The measurement data show that the VCSEL FM response follows an empirical square root law; i.e., the slope of the response above the cutoff frequency is $-1 / 2$ in a doubly logarithmic scale.

We apply a sinusoidal injection current with constant amplitude but variable frequency to the VCSEL laser and measure the extent of the sinusoidal wavelength modulation of the emitted light. The frequency is varied from $0.1 \mathrm{kHz}$ to $1 \mathrm{MHz}$ with a variable step size.

The measurements were taken with a WMS method ${ }^{10}$ and, as reference, a step-scan spectrometer. The WMS method allows an indirect determination of the sinusoidal wavelength variation amplitude via observing the narrowing of the measured second harmonic signal. The step-scan spectrometer Vertex 70 (Bruker) uses short-time Fourier transformation (STFT) getting time-resolved spectra with a minimum time resolution of $5 \mathrm{~ns}$. We used the STFT spectrometer to measure about 100 complete spectra at different times in one period of the modulation signal and determined the wavelength modulation amplitude by evaluating the shift of the emission peak.

The performance of the following two different VCSEL structures shown in Figs. 1 and 2 has been studied:

- InP-based VCSEL at $1853 \mathrm{~nm}$, which is packaged in a windowless TO-46 housing and placed on a thermoelectrically cooled laser mount. It can be used to detect $\mathrm{HCl}$, $\mathrm{H}_{2} \mathrm{O}$, and $\mathrm{CH}_{4} \cdot{ }^{11}$ The long-wavelength $(\lambda \geqslant 1.3 \mu \mathrm{m})$ InPbased VCSEL is described in detail in Ref. 6.

- GaAs-based VCSEL at $763 \mathrm{~nm}$ in a TO-5 housing with window and thermoelectric cooler. It can be used to detect $\mathrm{O}_{2}{ }^{12}$ The fabrication and performance of this kind of VCSEL is described in Ref. 13.

The heat sink temperature of both VCSELs is kept constant at about $32{ }^{\circ} \mathrm{C}$ by a laser controller (Profile LDC 200) 


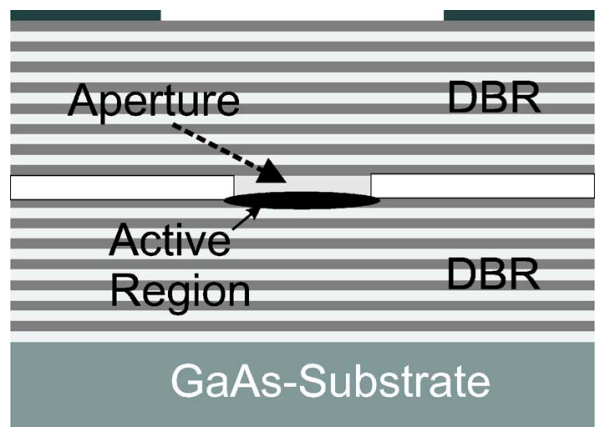

FIG. 1. (Color online) Schematical cross section of $\lambda=763 \mathrm{~nm}$ VCSEL. DBR: distributed Bragg reflector.

with a precision of $0.01{ }^{\circ} \mathrm{C}$. The current of the laser was controlled by a function generator (Agilent 33250A) with a shunt series resistor. The voltages and the resistance of the shunt were chosen so that a sinusoidal current with the amplitude 373 and $43 \mu \mathrm{A}$ was applied, respectively, as well as an appropriate bias current of 5.1 and $1.8 \mathrm{~mA}$, respectively. The amplitude of the sinusoidal current is small enough compared to the bias current to assure that the current to wavelength behavior is still linear.

The measured FM responses for both VCSELs are depicted in Figs. 3 and 4. It can clearly be seen that the frequency behavior of both VCSELs follows a square root law, since the slope reaches $-1 / 2$ in the doubly logarithmic scale (see dotted line in Figs. 3 and 4). An empirical model for the normalized FM response $|H(\omega)|$ is the following:

$$
|H(\omega)|=\frac{1}{\sqrt{1+\left(\omega / \omega_{c}\right)^{2 n}}},
$$

where $\omega_{c}$ is the $3 \mathrm{~dB}$ FM cutoff frequency and $-n$ is the slope in a doubly logarithmic scale for $\omega \rightarrow \infty$. The curve fit for the $763 \mathrm{~nm}$ VCSEL gives $n=0.52$, which is in very good agreement with the observed square root law. Although the slope parameter for large frequencies for the $1853 \mathrm{~nm}$ VCSEL is $n=0.65$, the measurement data show good agreement with the slope $-1 / 2$ in the measured frequency range as well.

The $3 \mathrm{~dB}$ cutoff frequencies $\omega_{c}$ are $\omega_{c} /(2 \pi) \approx 88 \mathrm{kHz}$ $(1853 \mathrm{~nm})$ and $\omega_{c} /(2 \pi) \approx 110 \mathrm{kHz}(763 \mathrm{~nm})$.

When we varied the amplitude of the sinusoidal injection current, the behavior of the normalized FM response did not change for both lasers.

We have carried out measurements of the FM response of two different VCSELs, based on different material systems and from different manufacturers. Although both show a low-pass behavior, it clearly does not correspond to a first

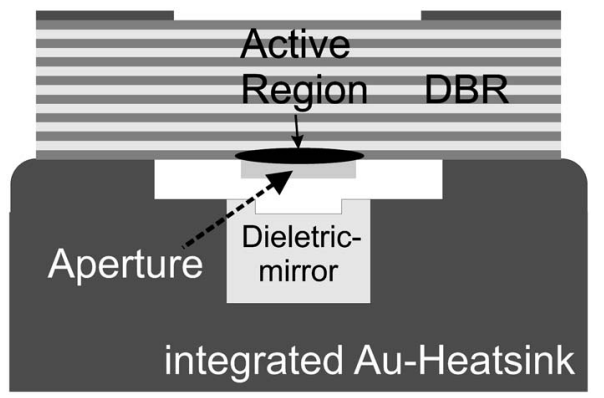

FIG. 2. Schematical cross section of $\lambda=1853 \mathrm{~nm}$ VCSEL. DBR: distributed Bragg reflector.

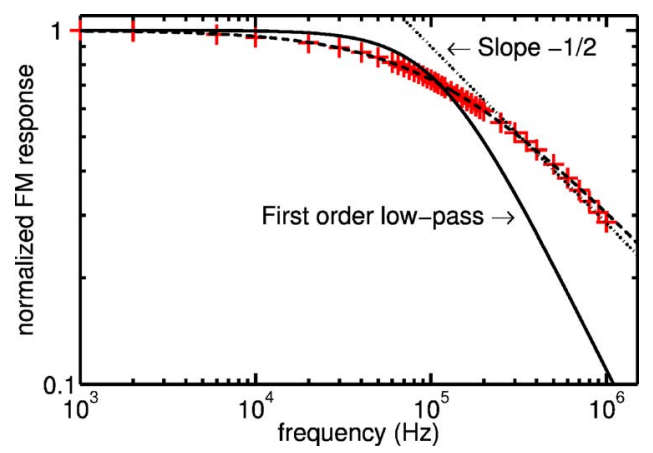

FIG. 3. (Color online) Measured FM response of the $763 \mathrm{~nm}$ VCSEL at $I_{\text {bias }}=1.8 \mathrm{~mA}$ and $I_{\text {mod }}=43 \mu \mathrm{A}$. The dotted and solid curves correspond to the slope of -0.5 and the first order low-pass case, respectively. The broken curve resembles the empirical model Eq. (1) with $n=0.52$.

order low pass, but follows a square root law above cutoff frequency (about $100 \mathrm{kHz}$ ). This means that doubling the frequency of the injection current will cause the current to wavelength tuning rate to drop only by about $30 \%(1 / \sqrt{2})$ instead of $50 \%$ as expected by the first order low pass. This is a clear advantage for wavelength modulation spectroscopy applications with VCSELs because the wavelength of the laser can be tuned at rather high frequencies without significant loss of the tuning efficiency compared to DFB lasers. For the $1853 \mathrm{~nm}$ VCSEL, the current to wavelength tuning rate of $0.6 \mathrm{~nm} / \mathrm{mA}$ at $1 \mathrm{kHz}$ drops only to $0.12 \mathrm{~nm} / \mathrm{mA}$ at $1 \mathrm{MHz}$. In contrast to VCSELs, the thermal behavior of DFB lasers has a much lower cutoff frequency of about several kilohertz ${ }^{9}$ and exhibits a slope of -1 in a logarithmic scaled FM response for high frequencies, although these lasers cannot accurately be described by a first order low pass either. Furthermore, for the same wavelength tuning range, the current consumption of the VCSEL is about one-tenth of the DFB lasers.

The empirical model Eq. (1) and the experimental data are in almost perfect agreement, whereby the exponent $n$ $\approx 0.5$ allows further adjustment of the exact form of the FM response.

Additionally, we can conclude that the step response of the VCSELs is not an exponential function as with a first order low pass. As a result, the FM cutoff frequency of the VCSELs cannot be determined easily by an exponential curve fit with a measurement of the wavelength step response.

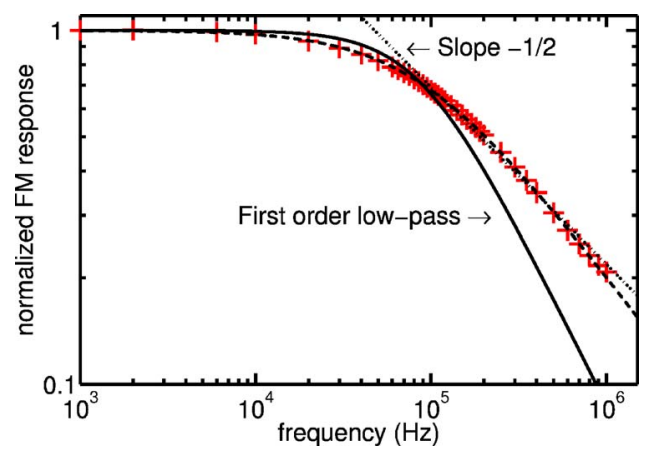

FIG. 4. (Color online) Measured FM response of the $1853 \mathrm{~nm}$ VCSEL at $I_{\text {bias }}=5.1 \mathrm{~mA}$ and $I_{\text {mod }}=373 \mu \mathrm{A}$. The dotted and solid curves correspond to the slope of -0.5 and the $3 \mathrm{~dB}$ low-pass case, respectively. The broken curve resembles the empirical model Eq. (1) with $n=0.65$. 
It is remarkable that in spite of their different device structures both VCSELs show nearly the same FM response. Obviously, the square root law behavior of the two different VCSELs is due to general thermal properties of the specific geometry of VCSELs. Apparently, the three-dimensional heat spreading in VCSELs is the principal reason for this behavior. Therefore, we conjecture that VCSELs with different structures will show a similar square root law behavior as well.

This work is funded by the project "NOSE" of the Federal Ministry of Education and Research of Germany (Contract No. 13N8772).

${ }^{1}$ H.-E. Li and K. Iga, Vertical-Cavity Surface-Emitting Laser Devices (Springer, Berlin, 2002), Vol. 1, pp. 1-3.

${ }^{2}$ F. Koyama, S. Kinoshita, and K. Iga, Appl. Phys. Lett. 55, 221 (1989).
${ }^{3}$ B. Weigl, M. Grabherr, R. Michalzik, G. Reiner, and K. J. Ebeling, IEEE Photonics Technol. Lett. 8, 971 (1996).

${ }^{4}$ M. Grabherr, R. Jaeger, R. Michalzik, B. Weigl, G. Reiner, and K. J. Ebeling, IEEE Photonics Technol. Lett. 10, 1304 (1997).

${ }^{5}$ M. Ortsiefer, R. Shau, G. Boehm, F. Koehler, and M.-C. Amann, Appl. Phys. Lett. 76, 2179 (2000)

${ }^{6}$ M.-C. Amann and M. Ortsiefer, Phys. Status Solidi A 203, 3538 (2006).

${ }^{7}$ R. Michalzik and K. J. Ebeling, Microelectron. Eng. 19, 123 (1992).

${ }^{8}$ M.-C. Amann and J. Buus, Tunable Laser Diodes (Artech House, Norwood, 1998), Vol. 1, pp. 94-99.

${ }^{9}$ S. Schilt and L. Thévenaz, Appl. Opt. 43, 4446 (2004).

${ }^{10}$ J. M. Supplee, E. A. Whittaker, and W. Lenth, Appl. Opt. 33, 6294 (1994).

${ }^{11}$ G. Totschnig, M. Lackner, R. Shau, M. Ortsiefer, J. Rosskopf, M.-C. Amann, and F. Winter, Meas. Sci. Technol. 14, 472 (2003).

${ }^{12}$ V. Weldon, J. O'Gorman, J. Pérez-Camacho, and J. Hegarty, Electron. Lett. 32, 219 (1996).

${ }^{13}$ M. Grabherr, D. Wiedenmann, R. Jaeger, and R. King, Proc. SPIE 5737, 120 (2005). 